\title{
Estimates and Asymptotic of Self-similar Solutions to a Nonlinear Filtration Problem with Variable Density and Nonlocal Boundary Conditions
}

\author{
Mirsaid Aripov, Zafar Rakhmonov* \\ Department of Informatics and Applied Programming, National University of Uzbekistan, Uzbekistan \\ *Corresponding Author: zraxmonov@inbox.ru
}

Copyright (C) 2016 by authors, all rights reserved. Authors agree that this article remains permanently

open access under the terms of the Creative Commons Attribution License 4.0 International License

\begin{abstract}
In this paper we study the global solvability or nosolvability of a nonlinear filtration problem with nonlinear flux boundary condition in the fast diffusion case. The critical global existence and critical Fujita exponent by constructing various self-similar supersolutions and subsolutions are obtained.
\end{abstract}

Keywords Self-similar, Global Solvability, Blow-up, Critical Exponent, Asymptotic Behavior, Numerical Analysis

\section{Introduction}

Consider the following polytrophic filtration equation in $(x, t) \in R_{+} \times(0,+\infty)$ with variable density

$$
\rho(x) \frac{\partial u}{\partial t}=\frac{\partial}{\partial x}\left(\left|\frac{\partial u^{m}}{\partial x}\right|^{p-2} \frac{\partial u^{m}}{\partial x}\right),
$$

subject to a nonlinear boundary flux

$$
-\left|\frac{\partial u^{m}}{\partial x}\right|^{p-2} \frac{\partial u^{m}}{\partial x}(0, t)=u^{q}(0, t), \quad t \in(0,+\infty)
$$

and initial value condition

$$
u(x, 0)=u_{0}(x), \quad x \in R_{+},
$$

where $\rho(x)=(1+x)^{n}, m>0, p>1, q>0, n \in R$ and $u_{0}(x)$ is a nontrivial, nonnegative, bounded and appropriately smooth function. In this paper we assume that $p$ satisfies a condition

$$
1<p<1+\frac{1}{m}
$$

Equation of the form (1) meets in various applications [1, $2,5,6,19,21,22]$. Equation (1) and its $N$-dimensional version arise in some physical models such as population dynamics, chemical reactions, heat transfer, etc [7]. In particular, equation (1) may be used to describe the no stationary flow in a porous medium of fluids with a power dependence of the tangential stress on the velocity of displacement under polytrophic conditions. In this case, equation (1) is called the non-Newtonian polytrophic filtration equation, which has been intensively studied (see [1-8] and references therein). The nonlinear boundary condition (2) can be used to describe the influx of energy input at the boundary $x=0$ [7]. For instance, in the heat transfer process (2) represents the heat flux, and hence the boundary conditions represent a nonlinear radiation law at the boundary. This kind of boundary condition appears also in combustion problems when the reaction happens only at the boundary of the container, for example because of the presence of a solid catalyzer, see [5] for a justification. Equation (1) is called a parabolic equation with variable density $[13,14]$. The particular feature of equation (1) is its power and gradient-dependent diffusivity.

In [6], Galaktionov and Levine studied the problem (1)-(3) for $p=2, n=0$ and for $m=1, n=0$. They proved that for the problem (1)-(3) the critical global exponent is $q_{0}=$ $(m+1) / 2$ and the critical Fujita exponent is $q_{c}=m+1$ (for $p=2, n=0$ ), while for the $m=1, n=0$ critical global exponent is $q_{0}=2(p-1) / p$ and the critical Fujita exponent is $q_{c}=2(p-1)$.

Wang and Yin [4], Li and Mu [7] studied problem (1)-(3) in the cases of a slow diffusion and a fast diffusion, respectively. They showed that the critical global existence exponent and critical Fujita exponent are $q_{0}=(m+1)(p-1) / p$ and $q_{c}=(m+1)(p-1)$.

Jiang and Zheng [8] studied the following problem:

$$
\left\{\begin{array}{l}
u_{t}=\left(\left|u_{x}\right|^{\beta}\left(u^{m}\right)_{x}\right)_{x}, \quad x>0, \quad 0<t<T, \\
-\left|u_{x}\right|^{\beta}\left(u^{m}\right)_{x}(0, t)=u^{p}(0, t), \quad 0<t<T \\
u(x, 0)=u_{0}(x), \quad x>0
\end{array}\right.
$$

where $m \geq 1, p>0, \beta>0$. They obtained the critical global existence exponent $p_{0}=(2 \beta+m+1) /(\beta+2)$ and the critical Fujita exponent $p_{c}=2 \beta+m+1$.

Many authors [9-11, 18, 19] intensively studied the Cauchy problem to the equation (1) in particular value of parameters. For instance Qi [9] studied the following Cauchy 
problem

$$
\begin{aligned}
& u_{t}=\Delta u^{m}+|x|^{\sigma} u^{p}, \quad x \in R^{N}, \quad t>0 \\
& u(x, 0)=u_{0}(x), \quad x \in R^{N},
\end{aligned}
$$

and established the critical Fujita exponent $p_{c}=m+$ $(2+\sigma) / N$ for $m>(N-2) / N$.

In [18], some Fujita type results extended to

$$
|x|^{m} u_{t}=\Delta u^{k}+|x|^{n} u^{q}, \quad x \in R^{N}, \quad t>0,
$$

with $q>k \geq 1$ and $0<m \leq n<q m+q-1$ and the critical Fujita exponent was given as $q_{c}=k+(2+n) /(N+m)$.

The Cauchy problem of another nonlinear diffusive equation of the form

$$
u_{t}=\operatorname{div}\left(|\nabla u|^{p-2} \nabla u\right)+|x|^{n} u^{q},
$$

where $p>(N+1) /(2 N), q>1$ was also considered by some authors. For the problem (4) with $p>2$ and $n=0$, Qi $[10,11]$ obtained that $q_{c}=p-1+p / N$ is the critical Fujita exponent of (4) and $q_{c}$ belongs to the blow-up case. If $n \neq 0$ in (4), Qi and Wang [12] proved that the critical Fujita exponent is $q_{c}=p-1+(p+n) / N$ for $(N+1) /(2 N)<$ $p<2$.

Zhongping Li, Chunlai Mu and Wanjuan Du [19] consider the positive solution to a Cauchy problem in $R^{N}$ of the fast diffusive equation:

$$
|x|^{m} u_{t}=\operatorname{div}\left(|\nabla u|^{p-2} \nabla u\right)+|x|^{n} u^{q}
$$

where $\frac{2 N+m}{N+m+1}<p<2,0<m \leq n<q m+N(q-1)$, with nontrivial, nonnegative initial data. They proved that $q_{c}=p-1+\frac{p+n}{N+m}$ is the critical Fujita exponent.

Regular property of the Cauchy problem for the equations

$$
s(x) \frac{\partial u}{\partial t}=\operatorname{div}\left(u^{k-1}|D u|^{\lambda-1} D u\right), \quad(x, t) \in R^{N+1}
$$

where $s(x)=|x|^{-l}, l \geq 0, D u \equiv\left(\frac{\partial u}{\partial x_{1}}, \ldots, \frac{\partial u}{\partial x_{N}}\right)$, was considered by authors of the work [20].

Martynenko and Tedeev [13, 14], studied the Cauchy problem for the following two equations with variable coefficients:

$$
\rho(x) u_{t}=\operatorname{div}\left(u^{m-1}|\nabla u|^{\lambda-1} \nabla u\right)+u^{p}, \quad x \in R^{N}, \quad t>0
$$

and

$\rho(x) u_{t}=\operatorname{div}\left(u^{m-1}|\nabla u|^{\lambda-1} \nabla u\right)+\rho(x) u^{p}, \quad x \in R^{N}, \quad t>0$ where $\lambda>0, m+\lambda-2>0, p>m+\lambda-1, \rho(x)=$ $|x|^{-n}$ or $\rho(x)=(1+|x|)^{-n}$. It was shown that under some restrictions on the parameters, any nontrivial solution to the Cauchy problem blows up in a finite time. Moreover, the authors established a sharp universal estimate of the solution near the blow-up point.

Authors of the work [17] investigated properties of weak solutions of the Cauchy problem for the following equation with variable coefficients:

$$
\rho_{1}(x) u_{t}=\operatorname{div}\left(|x|^{n} u^{m-1}|\nabla u|^{\lambda-1} \nabla u\right)+\rho_{1}(x) u^{p},
$$

where $\lambda>0, m+\lambda-2>0, p>m+\lambda-1, \rho_{1}(x)=|x|^{\delta-2}$.

Properties of the more general equation (1) with nonlocal nonlinear boundary condition were considered in [16] where were established the critical exponent of the Fujita type and second critical value.

This work is detailed and modified versions of the published work of the authors [23]. The main purpose of this paper is to study the conditions of solvability or non-solvability of the problem (1)-(3) on the basis of the self-analysis and the method of standard equations [2], to obtain the critical exponent of the global existence of solutions and the critical exponent Fujita type. The leading term of the asymptotic behavior of self-similar solutions of the problem (1)-(3) is obtained. On the basis of the asymptotic of solutions, suitable initial approximations are offered for the iterative process in the case of fast diffusion, depending on the values of the numeric parameters. At some points for the proof of Theorems 1-4 we used the terminology and develop methodology applied in $[1,2,4,7]$.

\section{Estimates of solutions}

Let's formulate the results on global solvability or nosolvability of the problem (1)-(3). We introduce the notation

$$
\begin{gathered}
q_{0}=\frac{(m(n+1)+1)(p-1)}{p+n}, q_{c}=m(p-1)+\frac{p-1}{n+1}, \\
Q_{\infty}=\left\{(t, x): 0<t<+\infty, x \in R_{+}\right\} .
\end{gathered}
$$

Theorem 2.1. If $0 \leq q \leq q_{0}$, then each solution of the problem (1)-(3) exists globally.

Proof. Let

$$
u_{+}(x, t)=e^{L t} g(\xi)
$$

with

$$
g(\xi)=\left(K+e^{-M \xi^{\frac{p+n}{p-1}}}\right)^{1 / m}, \xi=(1+x) e^{J t},
$$

where $L=J(p+n) /[1-m(p-1)], J=(K+1)^{2}, M=$ $(K+1)^{q /[m(p-1)]}$. A direct calculation yields

$$
-\left.\left|\frac{\partial u_{+}^{m}}{\partial x}\right|^{p-2} \frac{\partial u_{+}^{m}}{\partial x}\right|_{x=0}=-e^{J_{1} t}\left|\left(g^{m}\right)^{\prime}\right|^{p-2}\left(g^{m}\right)^{\prime}(1)
$$

$$
\frac{\partial}{\partial x}\left(\left|\frac{\partial u_{+}^{m}}{\partial x}\right|^{p-2} \frac{\partial u_{+}^{m}}{\partial x}\right)=e^{J_{2} t}\left(\left|\left(g^{m}\right)^{\prime}\right|^{p-2}\left(g^{m}\right)^{\prime}\right)^{\prime}(\xi),
$$

$$
(1+x)^{n} \frac{\partial u_{+}}{\partial t}=e^{(L-J n) t} \xi^{n}\left(L g(\xi)+J \xi g^{\prime}(\xi)\right) .
$$

where $J_{1}=(L m+J)(p-1), J_{2}=L m(p-1)+J p$. Note that $(L m+J)(p-1)-J n=L,(L m+J)(p-1) \geq q$, and hence, if

$$
\begin{gathered}
\left(\left|\left(g^{m}\right)^{\prime}\right|^{p-2}\left(g^{m}\right)^{\prime}\right)^{\prime}(\xi) \\
-\quad J \xi^{n+1} g^{\prime}(\xi) \\
-\quad L \xi^{n} g(\xi) \leq 0 \\
-\left|\left(g^{m}\right)^{\prime}\right|^{p-2}\left(g^{m}\right)^{\prime}(1) \geq g^{q}(1)
\end{gathered}
$$

then

$$
\rho(x) \frac{\partial u_{+}}{\partial t} \geq \frac{\partial}{\partial x}\left(\left|\frac{\partial u_{+}^{m}}{\partial x}\right|^{p-2} \frac{\partial u_{+}^{m}}{\partial x}\right)
$$




$$
-\left|\frac{\partial u_{+}^{m}}{\partial x}\right|^{p-2} \frac{\partial u_{+}^{m}}{\partial x}(0, t) \geq u_{+}^{q}(0, t) .
$$

It is not difficult to verify that if $K \geq\left\|u_{0}\right\|_{\infty}^{m}$ is large enough, then (5) and (6) are satisfied. Also, we have $u_{+}(x, 0) \geq$ $u_{0}(x)$ and $u_{+}(0,0)>u_{0}(0)$. Thus by comparison, we conclude that $u(x, t) \leq u_{+}(x, t)$, which implies that $u$ is global.

Theorem 2.2. If $q>q_{c}$ then the problem (1)-(3) admits global solutions with small initial data.

Proof. Theorem 2.2 is proved by the method of comparison of solutions. Equation (1) have self-similar solutions in the form of

$$
u_{+}(t, x)=(T+t)^{-\gamma} f(\xi), \quad \xi=(1+x)(T+t)^{-\sigma}
$$

where

$$
\begin{aligned}
\gamma & =\frac{p-1}{q(p+n)-(p-1)(m(n+1)+1)}, \\
\sigma & =\frac{q-m(p-1)}{q(p+n)-(p-1)(m(n+1)+1)} .
\end{aligned}
$$

Let's construct an suppersolution of the problem (1)-(3). The function $u_{+}(t, x)$ will be an suppersolution of the problem (1)-(3) if the function $f(\xi)$ according comparison principle satisfy the following inequalities $[4,6,7]$

$$
\begin{gathered}
\frac{d}{d \xi}\left(\left|\frac{d f^{m}}{d \xi}\right|^{p-2} \frac{d f^{m}}{d \xi}\right)+\sigma \xi^{n+1} \frac{d f}{d \xi}+\gamma \xi^{n} f \leq 0 \\
-\left|\left(f^{m}\right)^{\prime}\right|^{p-2}\left(f^{m}\right)^{\prime}(1) \geq f^{q}(1)
\end{gathered}
$$

Consider the function

$$
\bar{f}(\xi)=D\left(a+b \xi^{\frac{p+n}{p-1}}\right)^{-\frac{p-1}{1-m(p-1)}}
$$

where $b=\frac{1-m(p-1)}{m(p+n)} \sigma^{1 /(p-1)}, a$ is positive constant, and $D>0$ is to be determined. Then (8) and (9) are equivalent to

$$
\begin{aligned}
& \left(-(n+1) D^{m(p-1)-1} \sigma+\gamma\right)(a+b) \\
& +\left(D^{m(p-1)-1}-1\right) \frac{\sigma b(p+n)}{1-m(p-1)} \xi^{\frac{p+n}{p-1}} \leq 0 \\
& \quad \sigma(a+b)^{-\frac{(q-1)(p-1)}{1-m(p-1)}} \geq D^{q-m(p-1)}
\end{aligned}
$$

Then it is easy to see that under the condition of Theorem 2.2 and $D^{1-m(p-1)}<\frac{(q-m(p-1))(n+1)}{p-1}$ the inequalities (11) and (12) are holds.

In conclusion, we note that, if $u_{0}(x)<u_{+}(0, x)$ and $u_{0}(x)<u_{+}(0,0)$, by comparison principle, we conclude that $u(t, x) \leq u_{+}(t, x)$, which implies that the solution exists globally.

Theorem 2.3. If $q>q_{0}$, then the solution of the problem (1)-(3) with appropriately large initial data blows up in a finite time.

Proof. We seek a solution of (1)-(3) in the form

$$
u_{-}(x, t)=(T-t)^{-\gamma} \vartheta(\xi), \xi=(1+x)(T-t)^{-\sigma},
$$

where $\vartheta(\xi)$ satisfies

$$
\frac{d}{d \xi}\left(\left|\frac{d \vartheta^{m}}{d \xi}\right|^{p-2} \frac{d \vartheta^{m}}{d \xi}\right)-\sigma \xi^{n+1} \frac{d \vartheta}{d \xi}-\gamma \xi^{n} \vartheta=0
$$

and $\gamma, \sigma$ are given above numbers. Let

$$
\bar{\vartheta}(\xi)=(A-\xi)_{+}^{K},
$$

where $A>0, K>1 / m$. We show that the function $\bar{\vartheta}(\xi)$ is subsolution of (14). For this function $\bar{\vartheta}(\xi)$ according to the principles comparisons solutions must satisfy to the following inequalities [6]

$$
\begin{gathered}
\frac{d}{d \xi}\left(\left|\frac{d \bar{\vartheta}^{m}}{d \xi}\right|^{p-2} \frac{d \bar{\vartheta}^{m}}{d \xi}\right)-\sigma \xi^{n+1} \frac{d \bar{\vartheta}}{d \xi}-\gamma \xi^{n} \bar{\vartheta} \geq 0 \\
-\left|\left(\bar{\vartheta}^{m}\right)^{\prime}\right|^{p-2}\left(\bar{\vartheta}^{m}\right)^{\prime}(1) \leq \bar{\vartheta}^{q}(1) .
\end{gathered}
$$

A simple calculation yields

$$
\begin{gathered}
\bar{\vartheta}^{\prime}(\xi)=-K(A-\xi)^{K-1}, \\
\left(\bar{\vartheta}^{m}\right)^{\prime}(\xi)=-K m(A-\xi)^{K m-1}, \\
\left(\left|\left(\bar{\vartheta}^{m}\right)^{\prime}\right|^{p-2}\left(\bar{\vartheta}^{m}\right)^{\prime}\right)^{\prime}(\xi)=G_{1}(A-\xi)^{(K m-1)(p-1)-1},
\end{gathered}
$$

where $G_{1}=(K m)^{p-1}(K m-1)(p-1)$, then, from (15) and (16) we have the following inequality

$$
\begin{gathered}
G_{1}+\sigma K \xi^{n+1}(A-\xi)^{(1-K m)(p-1)+K}-\gamma \xi^{n}(A-\xi)^{G} \geq 0 \\
(K m)^{p-1}(A-1)^{(K m-1)(p-1)} \leq(A-1)^{K q} .
\end{gathered}
$$

where $G=K(m+1)-p(K m-1)$. Note that if $q>q_{0}$ then we get following inequality

$$
\sigma K \xi^{n+1}(A-\xi)^{(1-K m)(p-1)+K} \geq 0
$$

And it remains to show that inequality (17) is true and

$$
G_{1}-\gamma \xi^{n}(A-\xi)^{G} \geq 0
$$

It suffices to show that (18) holds for $\xi=n A /[K(m+1)-$ $p(K m-1)+n]$. Let $\xi=n A /[K(m+1)-p(K m-1)+n]$, then (18) can be written in the form

$$
(K m)^{p-1}(K m-1)(p-1) \geq \gamma\left(\frac{A n}{G+n}\right)^{n}\left(\frac{A G}{G+n}\right)^{G} .
$$

Hence, by Theorem 2.3 and by choosing

$$
\begin{aligned}
& (K m)^{\frac{p-1}{K q-(K m-1)(p-1)}}+1 \leq A \\
& \leq\left(\frac{G_{1}}{\gamma}\right)^{\frac{1}{G+n}}\left(\frac{G+n}{G}\right)^{\frac{G}{G+n}}\left(\frac{G+n}{n}\right)^{\frac{n}{G+n}},
\end{aligned}
$$

we verify that the inequalities (17) and (18) hold. Thus (13) is a weak subsolution of the problem (1)-(3). Then, by according to the comparing principle solutions $u(x, t)$ blows up in finite time $T<+\infty$. Note that the result of Theorem 2.3 contains the results of $[3,7]$ when $n=0$.

Theorem 2.4. If $q_{0}<q<q_{c}$, then each nontrivial solution of the problem (1)-(3) blows up in a finite time.

Proof. Consider the following Zeldovich - Barenblatt type solution of the equation (1):

$$
u_{1}(x, t)=(\tau+t)^{-\frac{n+1}{m(p-1)(n+1)+p-1}} H(\varsigma),
$$

$H(\varsigma)=\left(c_{1}+b_{1}|\varsigma|^{\frac{p+n}{p-1}}\right)_{+}^{-\frac{p-1}{1-m(p-1)}}, \varsigma=(1+x)(\tau+t)^{-\frac{1}{\mu}}$ 
where $\tau \geq 0, c_{1}>0, \mu=m(p-1)(n+1)+p-1$, $b_{1}=\frac{1-m(p-1)}{(p+n) m}\left(\frac{1}{\mu}\right)^{\frac{1}{p-1}}$. When $n=0$, this function is the same one as in $[3,4,7]$. It is easy to verify that $H^{\prime}(\varsigma)=0$ and for $\varsigma \in\{\varsigma>0 \mid H(\varsigma) \geq 0\} H(\varsigma)$ satisfies

$$
\frac{d}{d \varsigma}\left(\left|\frac{d H^{m}}{d \varsigma}\right|^{p-2} \frac{d H^{m}}{d \varsigma}\right)+\frac{1}{\mu} \varsigma^{n+1} \frac{d H}{d \varsigma}+\frac{n+1}{\mu} \varsigma^{n} H=0 .
$$

By using the well-known properties of weak solutions of the problem (1)-(3), we deduce that there exists $t_{0} \geq 0$ such that $u\left(0, t_{0}\right)>0$. Therefore, there exists sufficiently large $\tau>0$ and small $c_{1}>0$ such that for all $x>0$

$$
u\left(x, t_{0}\right) \geq u_{1}\left(x, t_{0}\right), x \in(0,+\infty)
$$

It is easy to verify that $u_{1}(x, t)$ is a subsolution of the problem (1)-(3) in $(0,+\infty) \times\left(t_{0},+\infty\right)$. From the comparison principle, we obtain that

$$
u(x, t) \geq u_{1}(x, t),(x, t) \in(0,+\infty) \times\left(t_{0},+\infty\right) .
$$

We declare that there exists $t_{*}>t_{0}$ and $T$ large enough that

$$
u_{1}\left(x, t_{*}\right) \geq u_{-}(x, 0), x \in(0,+\infty),
$$

where $u_{-}(x, t)$ is given by (13). A simple calculation shows that (19) is valid provided

$$
\begin{gathered}
\left(\tau+t_{*}\right)^{-\frac{n+1}{\mu}} \gg T^{-\gamma} \\
\left(\tau+t_{*}\right)^{-\frac{1}{\mu}} \ll T^{-\sigma} .
\end{gathered}
$$

Since $q<q_{c}$,

$$
\gamma /(n+1)>\sigma
$$

Therefore there exists $t_{*}>t_{0}$ and $T$ large enough that (20) and (21) are both valid. Thus

$$
u\left(x, t_{*}\right) \geq u_{1}\left(x, t_{*}\right) \geq u_{-}(x, 0), x \in(0,+\infty),
$$

which with the comparison principle implies that $u(x, t)$ blows up in a finite time.

\section{Asymptotic of solutions of self- similar problem}

The next stage of the investigation is to study the asymptotic behavior of self-similar solutions of the problem (1)-(3) to enable us to obtain numerical results. We show that the function (10) constructed by the method of standard equations [2] is the asymptotic of a self-similar problem (8), (9).

Theorem 3.1. Let $1<p<1+1 / m$. Then at infinity the solution of (8), (9) has the asymptotic

$$
f(\xi) \sim \varpi \bar{f}(\xi)
$$

where

$$
\varpi=\left(\frac{q(p+n)-(p-1)(m(n+1)+1)}{(q-m(p-1))(m(n+1)+1)(p-1)}\right)^{\frac{1}{m(p-1)-1}} .
$$

Proof. Theorem 3.1 is proved by the method used in the work [16]. The solution of (8) is sought in the form

$$
f=\bar{f}(\xi) w(\eta)
$$

where $\eta=\ln \left(a+b \xi^{\frac{p+n}{p-1}}\right)$, and at $\eta \rightarrow+\infty(\xi \rightarrow+\infty)$, and it allows to explore an asymptotic stability of the solution of (8), (9) at $\eta \rightarrow+\infty$. As a result according transformation (22) equation (8) is reduced to

$$
\begin{aligned}
& \frac{d}{d \eta} L w+\left(\frac{\phi_{1}(\eta)}{\phi_{2}(\eta)} Y_{1}-\frac{p-1}{r}\right) L w+\frac{\sigma}{Y} L w_{1} \\
& -\frac{\gamma w}{Y b(p+n)} \frac{\phi_{1}(\eta)}{\phi_{2}(\eta)}=0,
\end{aligned}
$$

where $L w=w^{r}\left|\frac{w}{r}-\frac{w^{\prime}}{p-1}\right|^{p-2}\left(\frac{w}{r}-\frac{w^{\prime}}{p-1}\right), L w_{1}=\frac{w}{r}-$ $\frac{w^{\prime}}{p-1}, \phi_{1}(\eta)=e^{\eta}, \phi_{2}(\eta)=\frac{e^{\eta}-a}{b}, Y=\frac{(b(p+n))^{p-1} m^{p-1}}{p-1}$, $Y_{1}=\frac{(n+1)(p-1)}{b(p+n)}, r=1 /[1-m(p-1)]$. Hence, analyzing the solutions of equation (23) using method of the work [2] we easily see that $w \rightarrow \varpi$ at $\eta \rightarrow+\infty$.

\section{Numerical analysis of solutions}

We note that due to nonuniqueness of solutions many different cases arises in the numerical study of the problem (1)-(3). Therefore, the question arises of selecting a good an initial approximation preserving properties of nonlinearity. Depending on the parameters of the equation, this difficulty is overcome by appropriate choice of initial approximations, which are taken as asymptotic formulas established above. On the basis of the above qualitative studies were produced the numerical calculations. The numerical results show quickly convergence of the iterative process to the solution of the problem Cauchy (1)-(3) due to the successful choice of the initial approximation. Below are given some results of numerical experiments for different values of the numerical parameters.

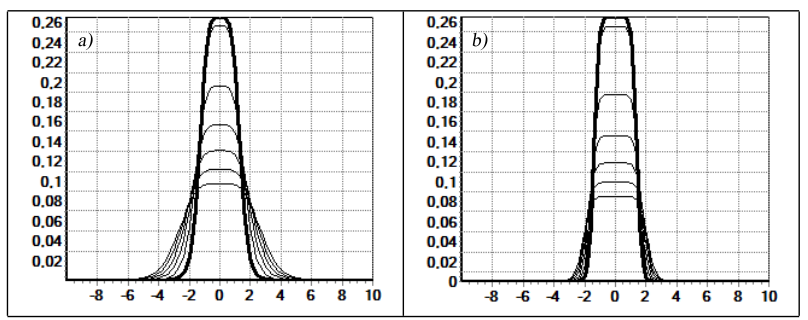

Figure 1. $\mathrm{m}=1.7, \mathrm{p}=1.45, \mathrm{q}=1.9$, a) $\mathrm{n}=0.5, \mathrm{~b}) \mathrm{n}=2$
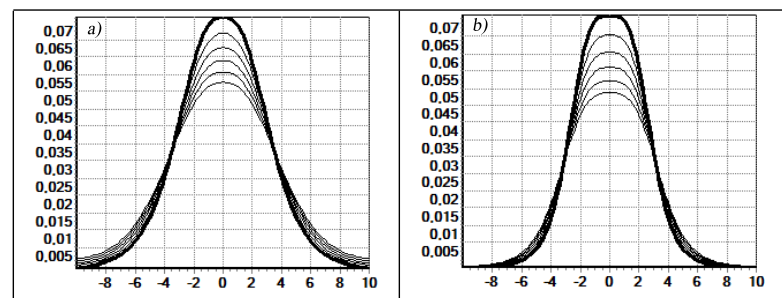

Figure 2. $\mathrm{m}=0.75, \mathrm{p}=1.78, \mathrm{q}=2$, a) $\mathrm{n}=0.3$, b) $\mathrm{n}=0.9$

In all figures bold lines corresponds to the initial approximations. Initial approximation is a very appropriate one and leads to the quick convergence. Results of numerical experiment and graphs shows that the self - similar solutions are very appropriate approximation. 


\section{REFERENCES}

[1] A.A.Samarskii, V.A.Galaktionov, S.P.Kurduomov (1987). A.P.Mikhajlov, Blow-up in quasilinear parabolic equations, .,Nauka (in Russian).

[2] M.Aripov (1988). Standard Equation's Methods for Solutions to Nonlinear problems (Monograph), Tashkent, FAN.

[3] C. Jin, J. Yin (2007). Critical exponents and non-extinction for a fast diffusive polytropic filtration equation with nonlinear boundary sources, Nonlinear Analysis, 67: 2217-2223.

[4] Z. Wang, J. Yin, C. Wang (2007). Critical exponents of the non-newtonianpolytropic filtration equation with nonlinear boundary condition, Applied Mathematics Letters, 20, 142 147.

[5] F.J. Mancebo, J.M. Vega (1999). A model of porous catalyst accounting for incipiently no isothermal effects, J. Differential Equations, 151, 79-110.

[6] V.A. Galaktionov, H.A. Levine (1996). On critical Fujita exponents for heat equations with nonlinear ?ux boundary condition on the boundary, Israel J. Math, 94, 125-146.

[7] Zhongping Li, Chunlai Mu (2008). Critical exponents for a fast diffusive polytropic filtration equation with nonlinear boundary flux, Mathematical Analysis and Applications, 346, 55-64.

[8] Z. X. Jiang and S. N. Zheng (2009). Doubly degenerate parabolic equation with nonlinear inner sources or boundary flux, Doctor Thesis, Dalian University of Tcchnology, In China.

[9] Y. W. Qi (1998). The critical exponents of parabolic equations and blow-up in $R^{N}$, Proc. Roy. Soc. Edinburgh Sect. A, 128(1), 123-136.

[10] Y. W. Qi (1995). Critical exponents of degenerate parabolic equations, Sci. China Ser. A, 38(10), 1153-1162.

[11] Y. W. Qi (1998). The global existence and nonuniqueness of a nonlinear degenerate equation, Nonlinear Analysis, 31(1-2), 117-136.

[12] Y. W. Qi and M. X. Wang (2002). Critical exponents of quasilinear parabolic equations, Mathematical Analysis and Applications, 267(1), 264-280.

[13] A.V. Martynenko and A. F. Tedeev (2007). The Cauchy problem for a quasilinear parabolic equation with a source and nonhomogeneous density, Comput. Math. Math. Phys., 2, 238-248.
[14] A.V. Martynenko and A. F. Tedeev (2008). On the behavior of solutions to the Cauchy problem for a degenerate parabolic equation with inhomogeneous density and a source, Comput. Math. Math. Phys., 48(7), 1145-1160.

[15] A.F. Tedeyev (2004). Conditions for the existence and nonexistence of a compact support in time of solutions of the Cauchy problem for quasilinear degenerate parabolic equations, Siberian Mathematical Journal, 45(1), 189-200.

[16] M.M. Aripov, Z.R. Rakhmonov (2013). Asymptotic behavior of self-similar solutions of a nonlinear problem polytropic filtration with a nonlinear boundary condition, Jour. Comp. Tech., 18(4), 50-55.

[17] Mersaid Aripov, Shakhlo A. Sadullaeva (2013). To properties of solutions to reaction-diffusion equation with double nonlinearity with distributed parameters, Jour. Sib. Fed. Univ. Math. Phys., 6(2), 157-167.

[18] Wanjuan Du and Zhongping Li (2013). Critical exponents for heat conduction equation with a nonlinear boundary condition, Int. Jour. of Math. Anal., 7(11), 517-524.

[19] Z. Li, Ch. Mu, and W. Du (2013). Critical Fujita exponent for a fast diffusive equation with variable coefficients, Bull. Korean Math. Soc., 50(1), 105-116.

[20] A.V. Martinenko, A.F. Tedeyev (2008). Regularity of solutions of degenerate parabolic equations with inhomogeneous density, Markov renewal equation, 5(1), 116-145.

[21] S.P. Kurdumov, E.S. Kurkina, G.G. Malinetskii (1979). Dissipative structures in environments with distributed parameters: M. IPMatem. USSR Academy of Sciences.

[22] M. Aripov, Z. Rakhmonov (2013). On the asymptotics of selfsimilar solution of a nonlinear heat conduction problem with variable density, DANRUz, 4, 3-5.

[23] M. Aripov, Z. Rakhmonov (2014). Numerical simulation of a nonlinear problem of a fast diffusive filtration with a variable density and nonlocal boundary conditions, Mathematics and Computers in Science and Engineering Series-23, 72-77.

[24] M. Aripov (2000). Asymptotic of the Solution of the NonNewton Polytropical Filtration Equation, ZAMM, 80(3), 767768 . 